\title{
ON NONLINEAR VARIATIONAL INEQUALITIES
}

\author{
E. TARAFDAR
}

\begin{abstract}
In this note we have given a direct proof of the result which states that if $K$ is a compact convex subset of a linear Hausdorff topological space $E$ over the reals and $T$ is a monotone and hemicontinuous (nonlinear) mapping of $K$ into $E^{*}$, then there is a $u_{0} \in K$ such that $\left(T\left(u_{0}\right), v-u_{0}\right)>0$ for all $v \in K$.
\end{abstract}

Introduction. Browder [1] has proved that if $K$ is a closed convex subset of a reflexive Banach space $E$ such that $0 \in K$ and $T$ is a monotone and hemicontinuous nonlinear mapping of $K$ into $E^{*}$ satisfying the coercivity condition, then there is a $u_{0} \in K$ such that $\left(T\left(u_{0}\right), v-u_{0}\right) \geqslant 0$ for all $v \in K$. Hartman and Stampacchia [3] have independently proved a similar result and made applications to second order nonlinear elliptic equations. This result with $c(u)=0$ (see Theorem 1.1 of [3]) is a special case of Browder's result [1]. With the closed convex subset $K$ of $E$ as assumed in [3], the coercivity condition on $T$ reduces the problem to proving the existence of $u_{0}$ satisfying the above inequality in a closed bounded convex subset of $K$ (see remark following Theorem 1.1 and Lemma 2.2 in [3]). Thus it is of interest to prove the above result in a weakly compact convex subset of an arbitrary Banach space. This would then contain Theorem 1.1 in [3] and the result of [1] as special cases. In fact the main object of this paper is to prove this result in a compact convex subset of a linear topological space over the reals without the coercivity condition on $T$. The techniques used in [1] and [3] are more or less the same, 'to prove the result in a finite dimensional case and then apply a limiting procedure'. We will give a direct proof of our result by applying a generalized version of a fixed point theorem of Browder [2].

The author is grateful to Dr. H. B. Thompson for discussions on this topic.

We first prove a slight generalization of a fixed point theorem of Browder [2, Theorem 1, p. 285] which will suit our purpose.

THEOREM 1. Let $K$ be a nonempty compact convex subset of a Hausdorff linear topological space $E$. Let $T$ be a multivalued mapping of $K$ into $2^{K}$ such that

(i) for each $x \in K, T(x)$ is a nonempty convex subset of $K$;

(ii) for each $y \in K, T^{-1}(y)=\{x \in K: y \in T(x)\}$ contains an open subset

Received by the editors December 7, 1976 and, in revised form, December 23, 1976.

AMS (MOS) subject classifications (1970). Primary 47H05; Secondary 47H10.

Key words and phrases. Variational inequality, monotone and hemicontinuous operators, fixed point theorem.

(C) American Mathematical Society 1977 
$O_{y}$ of $K\left(O_{y}\right.$ may be empty $)$

(iii) $\cup\left\{O_{y}: y \in K\right\}=K$.

Then there exists a point $x_{0} \in K$ such that $x_{0} \in T\left(x_{0}\right)$.

Proof. Although the proof is similar to that in [2], we include it for the sake of completeness. Since $K$ is compact, by (iii) there exists a finite family $\left\{y_{1}, y_{2}, \ldots, y_{n}\right\}$ such that $K=\cup_{i=1}^{n} O_{y_{i}}$. Let $\left\{f_{1}, f_{2}, \ldots, f_{n}\right\}$ be a partition of unity corresponding to this finite covering, i.e. each $f_{i}, i=1,2, \ldots, n$, is a real valued continuous function defined on $K$ such that $f_{i}$ vanishes outside $O_{y_{i}}, 0 \leqslant f_{i}(x) \leqslant 1$, for all $x \in K$ and $\sum_{i=1}^{n} f_{i}(x)=1$ for each $x \in K$.

We define a mapping $p: K \rightarrow K$ by

$$
p(x)=\sum_{i=1}^{n} f_{i}(x) y_{i}, \quad x \in K .
$$

Obviously $p$ maps $K$ into $K$ and is continuous. Also for each $k$ with $f_{k}(x) \neq 0$, $x \in O_{y_{k}} \subseteq T^{-1}\left(y_{k}\right)$, i.e. $y_{k} \in T(x)$. As $T(x)$ is convex, this implies that $p(x) \in T(x)$ for each $x \in K$.

Let $S$ be the finite dimensional simplex spanned by $y_{1}, y_{2}, \ldots, y_{n}$. Then clearly $p$ maps $S$ into $S$. Also, since $E$ is Hausdorff linear topological space, the topology on $S$ induced by the topology in $E$ is Euclidean. Hence by the Brouwer fixed point theorem, there is a point $x_{0} \in S$ such that $x_{0}=p\left(x_{0}\right) \in$ $T\left(x_{0}\right)$.

Let $K$ be a subset of a linear topological space $E$ over the reals and $T$ a single valued (nonlinear) mapping of $K$ into $E^{*}$. We say $T$ is monotone provided $(T(u)-T(v), u-v) \geqslant 0$ for all $u, v \in K$. Here $(\cdot, \cdot)$ denotes the pairing between $E^{*}$ and $E$.

$T: K \rightarrow E^{*}$ is said to be hemicontinuous if $T$ is continuous from the line segments in $K$ to the weak topology of $E^{*}$.

A point $u_{0} \in K$ is said to satisfy the variational inequality if

$$
\left(T\left(u_{0}\right), v-u_{0}\right) \geqslant 0 \text { for all } v \in K \ldots
$$

$u_{0}$ is also called a solution of (1).

LEMMA. If $K$ is a convex subset of a linear Hausdorff topological space $E$, and $T$ is a single valued mapping of $K$ into $E^{*}$ such that $T$ is monotone and hemicontinuous, then $u_{0}$ is a solution of (1) if and only if $u_{0}$ is a solution of

$$
\left(T(v), v-u_{0}\right) \geqslant 0 \text { for all } v \in K \ldots
$$

Proof. The proof of this lemma on a Banach space in [1, Lemma 1] or in [3, Lemma 2.3] also holds here. If $u_{0}$ satisfies (1), then an application of monotonicity shows that $u_{0}$ satisfies (2). Now suppose that $u_{0}$ satisfies (2). As in [1] and [3] we employ a device of Minty [4]. Let $v$ be an arbitrary point of $K$. Then since $K$ is convex, $v_{t}=(1-t) u_{0}+t v \in K$ for $0<t \leqslant 1$. By (2) we have 


$$
0 \leqslant\left(T\left(v_{t}\right), t\left(v-u_{0}\right)\right)=t\left(T\left(v_{t}\right), v-u_{0}\right) .
$$

Since $t>0,\left(T\left(v_{t}\right), v-u_{0}\right) \geqslant 0$.

Now letting $t \rightarrow 0$ and using hemicontinuity of $T, T\left(v_{t}\right) \rightarrow T\left(u_{0}\right)$ weakly in $E^{*}$. Hence $\left(T\left(u_{0}\right), v-u_{0}\right) \geqslant 0$.

REMARK. We note that in the proof of the first part the convexity of $K$ is not needed. In fact, if $T: K \rightarrow E^{*}$ is a monotone mapping of any set $K \subseteq E$ into $E^{*}$, then given $u \in K$, the set $\{v:(T(u), v-u) \geqslant 0\} \subseteq\{v:(T(v), v-$ $u) \geqslant 0\}$. This follows from the definition of monotonicity, i.e., $(T(v), v-u)$ $\geqslant(T(u), v-u)$.

THEOREM 2. Let $K$ be compact convex subset of a linear Hausdorff topological space $E$. Let $T$ be a (single valued) monotone (nonlinear) mapping of $K$ into $E^{*}$. Suppose further that

(*) for each $v \in K$ there exists $u \in K$ such that $(T(u), u-v)<0$.

Then there is a solution $u_{0}$ of (1), i.e. there is $u_{0} \in K$ such that $\left(T(u)_{0}, v-\right.$ $\left.u_{0}\right) \geqslant 0$ for all $v \in K$.

Proof. We assume that there is no solution of (1). Then for each $u \in K$, the set $\{v \in K:(T(u), v-u)<0\}$ is nonempty. We define a multivalued mapping $F: K \rightarrow 2^{K}$ by

$$
F(u)=\{v \in K:(T(u), v-u)<0\} .
$$

$F(u)$ is nonempty and clearly convex for each $u \in K$. We now consider

$$
F^{-1}(u)=\{v \in K: u \in F(v)\}=\{v \in K:(T(v), u-v)<0\} .
$$

For each $u \in K,\left[F^{-1}(u)\right]^{c}=$ the complement of $F^{-1}(u)$ in

$$
K=\{v:(T(v), u-v) \geqslant 0\} \subseteq\{v:(T(u), u-v) \geqslant 0\}
$$

by monotonicity of $T=B(u)$, say. Obviously $B(u)$ is a closed and convex subset of $K$. Thus the complement of $B(u)=[B(u)]^{c}$ is open in $K$. Since $\left[F^{-1}(u)\right]^{c} \subseteq B(u)$, it follows that $[B(u)]^{c} \subseteq F^{-1}(u)$. Thus for each $u \in K$, $F^{-1}(u)$ contains an open set $[B(u)]^{c}$ of $K$. Now from the hypothesis that for each $v \in K$, there exists $u \in K$ such that $(T(u), u-v)<0$, it follows that $\cup\left\{[B(u)]^{c}, u \in K\right\}=K$. Thus $F$ satisfies all the conditions of our Theorem 1. Hence there exists a point $w \in K$ such that $w \in F(w)$, i.e. $0>(T(w), w-$ $w)=0$, which is impossible.

Corollary. Let $K$ be a compact convex subset of a linear Hausdorff topological space E. Let $T$ be a monotone and hemicontinuous (nonlinear) mapping of $K$ into $E^{*}$. Then there is a solution $u_{0}$ of (1), i.e., there is $u_{0} \in K$ such that $\left(T\left(u_{0}\right), v-u_{0}\right) \geqslant 0$ for all $v \in K$.

Proof. If (*) of Theorem 2 holds, then we have a solution $u_{0}$ of (1) by Theorem 2. If $(*)$ does not hold, then it means precisely that there is $u_{0} \in K$ such that $\left(T(u), u-u_{0}\right) \geqslant 0$ for all $u \in K$. Since $T$ is hemicontinuous, the lemma implies that $\left(T\left(u_{0}\right), u-u_{0}\right) \geqslant 0$ for all $u \in K$, i.e. $u_{0}$ is a solution of the variational inequality. 
REMARK. It has already been pointed out in the introduction that our corollary contains the result of [1] and Theorem 1.1 of [3] as a special case. It is also worth noting that

(i) it follows from the proof of our theorem that we can replace the monotonicity condition by a weaker condition that for each $u \in K$, $\{v:(T(v), u-v) \geqslant 0\} \subseteq\{v:(T(u), u-v) \geqslant 0\}$;

(ii) in case of a locally convex Hausdorff topological space $E$, it does not matter whether we assume $K$ to be compact or weakly compact. The corollary still remains true as $T$ remains hemicontinuous in either case.

\section{REFERENCES}

1. F. E. Browder, Nonlinear monotone operators and convex sets in Banach spaces, Bull. Amer. Math. Soc. 71 (1965), 780-785.

2. The fixed point theory of multivalued mappings in topological vector spaces, Math. Ann. 177 (1968), 283-301.

3. P. Hartman and G. Stampacchia, On some non-linear elliptic differential-functional equations, Acta Math. 115 (1966), 271-310.

4. G. J. Minty, Monotone (non-linear) operators in Hilbert spaces, Duke Math. J. 29 (1962), 341-346.

Department of Mathematics, University of Queensland, St. Louis, Brisbane, QueensLAND, Australia 4067 